\title{
Role of CD319 Expression as A Diagnostic and Prognostic Marker in Plasma Cell Myeloma Patients Saad Sabry El-Osh ${ }^{1}$, Ayman Fathy Abd El-Halim², Shaimaa Saeed Ibrahim*1, Heba Allah Elsayed Abd El-Rhman ${ }^{1}$ \\ Departments of ${ }^{1}$ Clinical and Chemical Pathology and ${ }^{2}$ Internal Medicine, \\ Hematology Unit, Faculty of Medicine, Zagazig University, Egypt. \\ *Corresponding author: Shaimaa Saeed Ibrahim, Mobile: (+20)01006205976, Email: shaimaa.saeed39@ yahoo.com
}

\begin{abstract}
Background: The surface antigen CD319 (SLAMF7) is a marker of normal and malignant plasma cells in plasma cell myeloma. In view of the potential therapeutic use of SLAMF7-targeting antibodies, such as elotuzumab, the detection of SLAMF7 expression levels on abnormal plasma cells might play an important role in the evaluation of therapy responses. Objective: This study aimed to evaluate role of CD319 marker in diagnosis and prognosis of plasma cell myeloma patients. Patients and Methods: The study was Cohort study conducted on newly diagnosed plasma cell myeloma patients in Clinical Pathology Department and Internal Medicine Department-Hematology Unit at Zagazig University Hospitals in period between February 2019 and February 2021. It included 18 cases diagnosed according to clinical, pathological, morphological and immunophenotypic criteria. Follow up of patients was carried out after 3 months of initiation of treatment to assess response to therapy. Results: This study showed that CD319 (SLAMF7, CRACC, CS1) is a stable marker with high expression mean fluorescence intensity (MFI) on normal and malignant plasma cells. In this study, CD319 is found positive in all plasma cell myeloma (PCM) patients. Low expression of CD319 (if MFI below 128.3) was associated with a significant better response to treatment and good prognosis but high expression of CD319 was accompanied by bad response to treatment. It can be effectively used as a diagnostic and prognostic marker for PCM.

Conclusion: CD319 is found positive in all PCM patients. It can be effectively used as a diagnostic marker for PCM. Low expression of CD319 is associated with a significant better response to treatment and good prognosis. This indicates importance of CD319 as a prognostic marker of PCM.
\end{abstract}

Keywords: Bone marrow aspiration, CD319, Flow cytometry, Plasma cell myeloma.

\section{INTRODUCTION}

Plasma cell myeloma (PCM) is a bone marrowbased multifocal neoplastic proliferation of plasma cells, usually associated with an $\mathrm{M}$ protein in serum with evidence of organ damage related to the plasma cell neoplasm. It is associated with significant morbidity due to its end-organ destruction. The disease spans a clinical spectrum from asymptomatic to aggressive forms due to deposition of abnormal immunoglobulin chain in the tissues. The diagnosis of plasma cell myeloma is based on a combination of pathological, radiological and clinical features ${ }^{(\mathbf{1})}$.

PCM comprise about $1 \%$ of malignant tumors, 10 $15 \%$ of hematopoietic neoplasms, and $20 \%$ of deaths from hematological malignancies. It is more common in men than in women, with a male to female ratio of 1.1: 1 . The incidence of plasma cell myeloma increases progressively with patient age thereafter, with about $90 \%$ of cases occurring in patient aged more than 50 years, with the median age at diagnosis is 70 years $^{(2)}$.

CD319 marker, known as SLAM (Signaling lymphocytic activation molecule) family member 7 , is a protein that found in humans encoded by the SLAMF7 gene. It is located on chromosome 1 on long arm (1q23.3). It is a marker of normal and malignant plasma cells in plasma cell myeloma. In addition to PCs, SLAMF7 expression is found on other immune cells such as NK cells, NK-like T-cells, and CD8+ T-cells. In contrast to CD138 (the traditional plasma cell marker), CD319/SLAMF7 is much more stable and allows proper isolation of malignant plasma cells from delayed or even cryopreserved samples ${ }^{(3)}$. So CD319 expression (SLAMF7) plays an important role in diagnosis of plasma cell myeloma. In view of the potential therapeutic use of SLAMF7-targeting antibodies, such as elotuzumab, the detection of SLAMF7 expression levels on abnormal plasma cells might play an important role in the evaluation of therapy responses.

This study aimed to evaluate role of CD319 marker in diagnosis and prognosis of plasma cell myeloma patients and to correlate between CD319 marker and response to therapy.

\section{PATIENTS AND METHODS}

This study was carried out at Clinical Pathology Department and Internal Medicine DepartmentHematology Unit at Zagazig University Hospitals.

It included 18 cases (11 males and 7 females) with a male to female ratio of 1.5:1. Mean age of cases was $53 \pm 9.8$ years. 
Inclusion criteria: Newly diagnosed patients with plasma cell myeloma. Patients who had any plasma cell myeloma. Patients under treatment and follow up. Other malignancy and autoimmune disease.

\section{Ethical approval:}

The study was accepted by the Faculty of Medicine's Ethical Review Committee at Zagazig University. Every patient signed an informed written consent for acceptance of the study. It was in compliance with the World Medical Association's Code of Ethics (Helsinki Declaration), for human studies.

All patients were subjected to full history taking, full clinical examination. Laboratory investigations; Complete blood count (CBC) using sysmex XP Japan, with examination of Leishman stained PB smears for differential leucocytes count. The samples were obtained during the course of routine analysis and collected in EDTA anticoagulant tubes. Liver, kidney function tests, serum calcium and LDH (lactic acid dehydrogenase) using automated analyzer "Cobas 8000 platform-702c module, China". Also ESR (erythrocyte sedimentation rate) using automated analyzer "VISION-B, China".

Myeloma specific tests; Serum protein electrophoresis and immunofixation. Serum B2 microglobulin. Bence Jones protein in urine. Bone marrow aspiration and examination of Leishman stained smears to detect the percentage of bone marrow (BM) plasma cells. Also bone marrow biopsy film stained with H\&E. Skeletal bone survey including plain $\mathrm{x}$-ray. Multicolor flow cytometric immunophenotyping by using a panel of monoclonal antibodies against CD56, CD19, CD20, CD138, CD38, and CD45 using FACS Calibur Flow Cytometry, Becton-Dickinson (BD), USA. Detection of CD319 marker (CS1, SLAMF7, CRAAC) by using multicolor flow cytometry and its mean fluorescence intensity (MFI).

For MCF analysis, $100 \mu 1$ of blood count adjusted anticoagulated sample was used. First add lyse then red blood cells were lysed and washed twice with PBS. The supernatant was discarded and the cell pellet was suspended in PSB. Bone marrow sample was incubated with monoclonal antibodies in the dark at room temperature for $15 \mathrm{~min}$. After incubation, stained cells were quickly detected and analyzed using MCF (FACS Calibur Becton Dickinson, San Joe USA) Cell Quest software BD Bioscience, FACS Calibur 4 color using the following panel of cluster of differentiation (CD) antigens: CD138 PerCP, CD38 FITC, CD45 APC, CD54 PE, CD19 PerCP, CD20 FITC, CD319 PE, $к$ and $\lambda$ light-chain Ig (PE and FITC respectively) to confirm clonality. For identification of PCs at least 30,000 cells were required, cells were considered positive for a marker when more than $20 \%$ of cells expressed that $\operatorname{mark}^{(4)}$.

Identification of PCs by CD 38, CD138 occurs after setting a first $\mathrm{PC}$ gate in a CD38 vs CD138 bivariate dot plot. It was used as the reference PC gating approach and considered as the $100 \%$ threshold for PC identification and for further comparison of the performance of CD319 gating in combination with CD38, CD138, or 56. In case of compromised /low expression of CD138, PCs were detected through the use of the other PC markers present in the tube. Expression levels of individual markers were reported as mean fluorescence intensity values (MFI).

CD319 expression level was assessed as the mean fluorescence intensity, defined as mean of the fluorescence intensity of CD319-PE-positive PCs, using the Infinicyt software (Cytognos, Salamanca, Spain)

To ensure importance of CD319 in prognosis of PCM, we follow up PCM patients three months later after initiation of treatment. Then assess response of PCM patients to treatment.

In this study all PCM patients receive three cycles of chemotherapy during course of treatment. They receive VRd, bortezomib in day $1,8,15$, and 22, lenalidomide in day 1-21 (one week off) and dexamethasone in day $1,2,8,9,15,16,22$, and 23 for each cycle, then start another cycle. Almost all patients received biphosphate therapy (zometa) due to bone lesions.

After the third cycle re-evaluation of cases occur by CBC, serum B2 microglobulin, serum protein electrophoresis and immunofixation, BMA and BM biopsy. Then we assessed response to treatment according to National Comprehensive Cancer Network $(\mathrm{NCCN}){ }^{(5)}$ regarding revised IMWG response criteria ${ }^{(6)}$.

\section{Statistical analysis}

The collected data were coded, processed and analyzed using the SPSS (Statistical Package for Social Sciences) version 22 for Windows ${ }^{\circledR}$ (IBM SPSS Inc, Chicago, IL, USA). Data were tested for normal distribution using the Shapiro Walk test. Qualitative data were represented as frequencies and relative percentages. Chi square test $(\chi 2)$ to calculate difference between two or more groups of qualitative variables. Quantitative data were expressed as mean \pm SD (Standard deviation). Independent samples t-test was used to compare between two independent groups of normally distributed variables (parametric data). $\mathrm{P}$ value $<0.05$ was considered significant.

\section{RESULTS}

The demographic and basic characteristics data of PCM patients are shown in table 1. 
Table 1: The demographic and characteristic data of PCM patients

\begin{tabular}{|l|c|c|}
\hline Variable & \multicolumn{2}{|c|}{ N=18 } \\
\hline Age (years) & \multicolumn{2}{|c|}{$\mathbf{5 3 \pm 9 . 8}$} \\
Mean \pm SD & \multicolumn{2}{|c|}{$\mathbf{3 1 - 6 5}$} \\
Range & No. & \% \\
\hline & 11 & 61.1 \\
\hline Sex: & 7 & 38.9 \\
Male & Mean \pm SD & Median \\
Female & $6.99 \pm 0.9$ & 7.1 \\
\hline Variable & $9.4 \pm 1.3$ & 9 \\
\hline TLC & $205.4 \pm 9.9$ & 30 \\
\hline Hemoglobin (Hb) & $27.8 \pm 1.8$ & 1 \\
\hline Platelets & $1.8 \pm 0.2$ & 10.6 \\
\hline BM plasma cell (\%) & $10.4 \pm 0.3$ & 3.25 \\
\hline Creatinine & $2.9 \pm 0.2$ & 180 \\
\hline Ca & $193.6 \pm 7.8$ & 155 \\
\hline Albumin & $159.7 \pm 3.1$ & 208 \\
\hline B2 microglobulin & & \\
\hline LDH & & \\
\hline
\end{tabular}

BM: Bone marrow, LDH: Lactic acid dehydrogenase

Table 2 shows that as regard M protein, half of them (50\%) was IgG kappa. As regard bone lesion, 88.95 of them were multiple.

Table 2: M protein and bone lesion among PCM patients

\begin{tabular}{|l|c|c|}
\hline \multicolumn{1}{|c|}{ Variable } & $\mathbf{N = 1 8}$ & $\mathbf{\% = 1 0 0}$ \\
\hline M protein: & 9 & \\
- IgG kappa & 6 & 50 \\
- IgG lambda & 3 & 33.3 \\
- IgA lambda & & 16.7 \\
\hline Bone lesion: & 16 & \\
- Multiple & 2 & 88.9 \\
- Solitary & & 11.1 \\
\hline
\end{tabular}

There was highly statistically significant association between CD319\% and response to treatment (table 3).

Table 3: Association between CD319\% and response to treatment among the studied group

\begin{tabular}{|l|r|c|c|c|}
\hline \multirow{2}{*}{\begin{tabular}{l} 
Variable \\
CD319\%: \\
\cline { 2 - 4 }
\end{tabular}} & \multicolumn{3}{|c|}{ Response to treatment } & \multirow{2}{*}{ P-value } \\
\hline
\end{tabular}

HS: Highly significant.

CR: complete response, PR: partial response, PD: progressive disease

On using receiver operating characteristic (ROC) curve, cutoff point was 128.3 (Table 4).

Table 4: ROC curve for CD319 MFI in plasma cell myeloma

\begin{tabular}{|c|c|c|c|c|}
\hline Variable & AUC & Cutoff point & Sensitivity & Specificity \\
\hline MFI & 0.64 & 128.3 & 90 & 66 \\
\hline
\end{tabular}

AUC: Area under the curve.

There was statistically significant correlation between high expression of CD319 and low hemoglobin concentration of plasma cell myeloma patients. In addition there was highly statistically significant correlation between high expression of CD319 and high expressions of CD138/38 and CD56. 
Table 5: Correlation between expression of CD319 MFI, demographic and characteristic data of PCM patients

\begin{tabular}{|c|c|c|c|}
\hline \multirow[t]{2}{*}{ Variable } & \multicolumn{2}{|c|}{ Expression of CD319 MFI } & \multirow[t]{2}{*}{ P-value } \\
\hline & $\begin{array}{c}\text { Low expression } \\
(\mathrm{N}=11)\end{array}$ & $\begin{array}{c}\text { High expression } \\
(\mathrm{N}=7)\end{array}$ & \\
\hline $\begin{array}{l}\text { Age (years): } \\
\quad \text { Mean } \pm \text { SD }\end{array}$ & $51.9 \pm 1.5$ & $54.7 \pm 6.9$ & 0.57 \\
\hline $\begin{array}{l}\text { Sex: } \\
\text { Male } \\
\text { Female } \\
\end{array}$ & $\begin{array}{l}7(63.6) \\
4(36.4)\end{array}$ & $\begin{array}{l}4(51.7) \\
3(42.9)\end{array}$ & 1 \\
\hline $\begin{array}{ll}\text { TLC: } & \text { Mean } \pm \text { SD }\end{array}$ & $7.1 \pm 0.2$ & $6.9 \pm 0.9$ & 0.679 \\
\hline $\begin{array}{l}\text { Hemoglobin: } \\
\text { Mean } \pm \text { SD }\end{array}$ & $10.5 \pm 0.3$ & $7.8 \pm 0.2$ & $0.012 *(\mathrm{~S})$ \\
\hline $\begin{array}{l}\text { Platelets: } \\
\qquad \text { Mean } \pm \text { SD }\end{array}$ & $229.7 \pm 8$ & $167.3 \pm 7.1$ & 0.108 \\
\hline $\begin{array}{c}\text { BM plasma cell }(\%): \\
\text { Mean } \pm \text { SD }\end{array}$ & $30.5 \pm 2.5$ & $26 \pm 3.5$ & 0.403 \\
\hline Ca: $\quad$ Mean \pm SD & $10.5 \pm 0.6$ & $10.2 \pm 0.78$ & 0.715 \\
\hline $\begin{array}{l}\text { Creatinine: } \\
\quad \text { Mean } \pm \text { SD }\end{array}$ & $2.2 \pm 0.6$ & $1.1 \pm 0.05$ & 0.325 \\
\hline $\begin{array}{l}\text { Albumin: } \\
\qquad \text { Mean } \pm \text { SD }\end{array}$ & $3.3 \pm 0.08$ & $2.2 \pm 0.5$ & 0.057 \\
\hline $\begin{array}{l}\text { B2 microglobulin: } \\
\text { Mean } \pm \text { SD }\end{array}$ & $197.3 \pm 1.4$ & $187.7 \pm 5.9$ & 0.715 \\
\hline LDH: $\quad$ Mean \pm SD & $162.6 \pm 1.9$ & $155 \pm 7.1$ & 0.726 \\
\hline $\begin{array}{ll} & \text { M protein: } \\
- & \text { IgG kappa } \\
\bullet & \text { IgG lambda } \\
\bullet & \text { IgA lambda } \\
\end{array}$ & $\begin{array}{l}4(36.4) \\
4(36.4) \\
3(27.3)\end{array}$ & $\begin{array}{c}5(71.4) \\
2(28.6) \\
0(0)\end{array}$ & 0.256 \\
\hline $\begin{array}{ll} & \text { Bone lesion: } \\
\text { - } & \text { Multiple } \\
\text { - } & \text { Solitary }\end{array}$ & $\begin{array}{l}9(81.8) \\
2(18.2)\end{array}$ & $\begin{array}{c}7(100) \\
0(0)\end{array}$ & 0.497 \\
\hline $\begin{array}{l}\text { CD138/38 \%: } \\
\text { Mean } \pm \text { SD }\end{array}$ & $29.5 \pm 2.9$ & $47 \pm 1.9$ & $\begin{array}{l}<0.001 * \\
\text { (HS) }\end{array}$ \\
\hline $\begin{array}{l}\text { CD56 \%: } \\
\text { Mean } \pm \text { SD }\end{array}$ & $24.8 \pm 2.1$ & $34.3 \pm 2.7$ & $\begin{array}{l}<0.001 * \\
\text { (HS) }\end{array}$ \\
\hline $\begin{array}{l}\text { CD } 319 \%: \\
\text { Mean } \pm \text { SD }\end{array}$ & $58.9 \pm 4.5$ & $91.8 \pm 3.7$ & $\begin{array}{l}<0.001 * \\
\text { (HS) }\end{array}$ \\
\hline
\end{tabular}

TLC: Total: Leucocytic count, BM: Bone marrow, LDH: Lactic acid dehydrogenase

*: Significant difference, S: Significant, HS: Highly significant

Figure 1 and 2 showed positive CD138/38, positive CD56/CD138 and positive CD319/CD138 in the same sample of the PCM patient for each figure separately. 


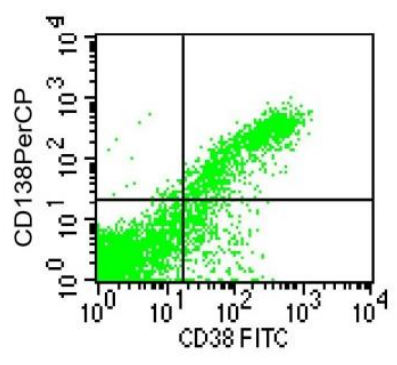

$1(A)$

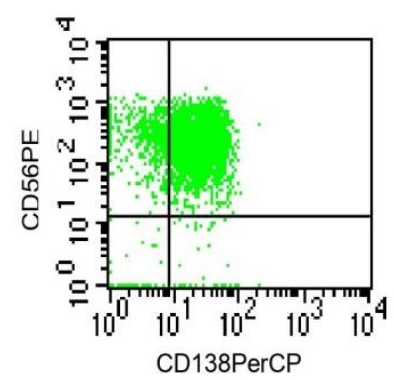

1 ( B )

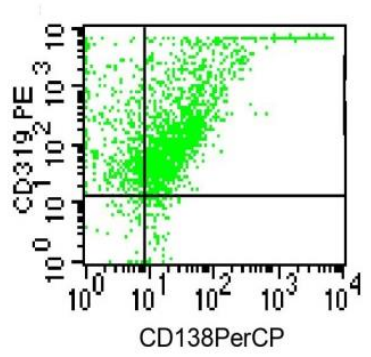

$1(\mathrm{C})$

Figure (1): Immunophenotyping of plasma cells from BM aspirate of PCM patient revealed:

A- Positive CD138/38

B- Positive CD56/CD138

C- Positive CD319/CD138

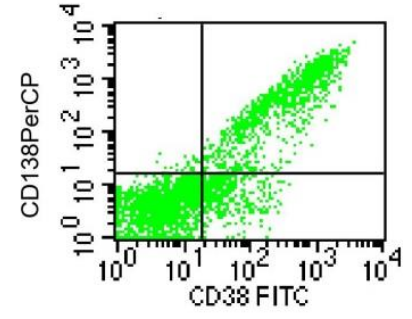

$2(A)$

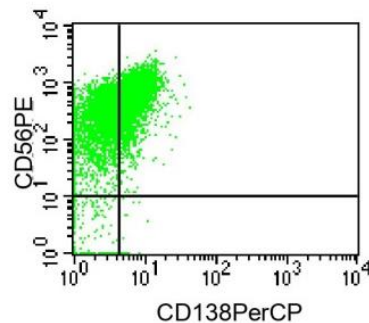

$2(B)$

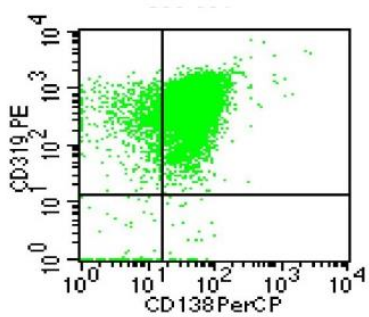

$2(\mathrm{C})$

(2): Another immunophenotyping of plasma cells from BM aspirate of PCM patient revealed:

Figure

A- Positive CD138/CD38

B- Positive CD56/CD138

C- Positive CD319/CD138

There was statistically significant association between expression of CD319 and response to treatment. As low expression of CD319 is associated with a significant better response to treatment (Table 6).

Table 6: Association between expression of CD319 MFI and response to treatment among the studied group

\begin{tabular}{|c|c|c|c|c|c|}
\hline \multirow{2}{*}{ Variable } & \multicolumn{3}{|c|}{ Expression of CD319 MFI } & \multirow{2}{*}{ P-value } \\
\cline { 2 - 5 } & \multicolumn{2}{|c|}{ Low expression } & \multicolumn{2}{c|}{ high expression } & \\
\cline { 2 - 5 } & N=11 & $\%$ & N=7 & $\%$ & \multirow{2}{*}{0.07} \\
Response to treatment: & & & & & \\
$\bullet$ CR & 4 & 36.4 & 0 & 0 & \\
$\bullet$ PR & 7 & 63.6 & 5 & 71.4 & \\
PD & 0 & 0 & 2 & 28.6 & \\
\hline
\end{tabular}

CR: complete response, PR: partial response, PD: progressive disease 


\section{DISCUSSION}

This study showed that the mean age of PCM patients is 53 years and the majority of them were males $(61.1 \%)$ with a male to female ratio $1.5: 1$. While in the study done by Bouatay et al. ${ }^{(7)}$ the mean age of cases was 67 years with a male to female ratio 1.7:1. In addition, Pojero et al. ${ }^{(8)}$ found that mean age of PCM patients was 60 years and the majority of them were males $(56 \%)$ with a male to female ratio 1.2:1.

In the present study as regard $M$ protein, half of PCM patients (50\%) was IgG kappa, 33.3\% of them were IgG lambda and $16.7 \%$ of them were IgA lambda. These results agree with the previous studies done by Bouatay et al. (7) and Aita et al. ${ }^{(9)}$ that indicate predominance of IgG kappa in multiple myeloma (MM) Patients.

Despite the presence of hypercalcemia, elevated creatinine and bone lesions that represent myeloma defining events, none of these clinical variables had any significant association with positive CD319 and its expression in our study. These findings are in concordance with Ishibashi $\boldsymbol{e t} \boldsymbol{a l}{ }^{(\mathbf{1 0})}$ who found that CD319 was not correlated with any clinical variables.

In the present study, CD319 (SLAMF7) levels were not correlated with the percentages of BM plasma cells. These results are potentially similar to those reported by Ishibashi et al. ${ }^{(10)}$ who found that soluble SLAMF7(sSLAMF7) levels were not correlated with the percentages of BM plasma cells and mRNA levels of SLAMF7 in BM plasma cells from PCM patients.

Regarding laboratory variables, positive CD319\% and MFI were not found to be statistically significantly associated with any of them, except for hemoglobin $(\mathrm{Hb})$ and multiple myeloma immunophenotypic markers. $\mathrm{Hb}$ exhibited a statistically significant negative correlation as high expression of CD319 associated with low $\mathrm{Hb}$ concentration of plasma cell myeloma patients but there was a highly statistically significant strong positive correlation between CD319 and its expression and CD138/38, CD 56. This is in concordance with a study done by Flores-Montero et al. ${ }^{(11)}$, who found that there is positive correlation between CD138/38 and CD56 and CD319 .

In addition to CD138 and CD38, a few other PC associated markers have emerged as a potential candidates for MCF identification of BM PCs in MM. Among other markers, CD54, CD229, and CD319 have been identified as PC-associated markers and candidates to potentially replace CD38 and/or CD138 in the identification of BMPCs in PCM patients ${ }^{(8)}$.

CD319 plus CD38 combinations may be considered as robust alternatives to CD38 vs CD138 for the initial identification of both normal plasma cells (nPCs) and abnormal plasma cells in BM in PCM. This might be of great utility in some PCM cases, particularly in those showing loss or down regulation of CD138 due to delayed sample arrival or in case of frozen samples ${ }^{(\mathbf{3})}$.

Over all, our results show that CD319 (SLAMF7, CRACC, CS1) is a stable marker with high expression (MFI) in normal and malignant plasma cells. In our study, CD319 is found positive in all PCM patients. It can be effectively used as a diagnostic marker for PCM. This is in concordance with a study done by Sriram et al. ${ }^{(12)}$, who found that CD319 is stable immunophenotypic marker with strong, homogenous expression in PCs and can be effectively used as a gating marker in addition to traditional markers for plasma cell quantitation, especially after daratumumab-therapy.

Also our results agree with Veillette and Guo ${ }^{(13)}$ study. Their study shows that CD319 was expressed at high levels on myeloma PCs, independently of their cytogenetic/molecular back ground, which led Frigyesi et al. ${ }^{(3)}$, to propose CD319 to be more robust than CD138 for the isolation of BM PCs.

As regard results of CD319 and its high expressions on plasma cells, these are potentially similar to those reported by Touzeau et al. ${ }^{(14)}$, who found that high expression levels of SLAMF7 were observed in PCM patients including those with highrisk and low risk molecular profiles and with and without cytogenetic abnormalities.

Recently Soh et al. ${ }^{(15)}$, proved that CD319 is a suitable alternative to $\mathrm{CD} 38$ for identifying plasma cells also CD319 may be used alone or in combination with CD38 to detect PCs in daratumumab or elotuzumab treated patients. To further elucidate the prognostic significance and the value of CD319 in determining outcome, CD319 and its expression (MFI) were studied in relation to treatment response. There was highly statistically significant association between CD 319 percentage and its expression (MFI) and response to treatment.

In our study as regard response to treatment in PCM patients, high expression of CD319 (above cut off point 128.3) including PD and PR associated with a significant bad response to treatment and bad prognosis. This is in concordant with study carried by Ishibashi et al. ${ }^{(10)}$ who showed for the first time that PCM patients with high soluble SLAMF7 (sSLAMF7) levels had shorter progression free survival (PFS) times and sSLAMF7 in serum of PCM patients may be a useful indicator of disease progression.

In addition, low expressions of CD319 including $\mathrm{CR}$ and/or PR were associated with a significant better response to treatment (good prognosis) and this agree with Ishibashi et al. ${ }^{\left({ }^{10)}\right.}$ study. They found that soluble SLAMF7 (sSLAMF7) levels in serum from MM 
patients were undetected or decreased when achieving CR or PR after antimyeloma therapy. But in contrast to that, Lisenko et al. ${ }^{(16)}$ who found that the mean fluorescence intensity analysis revealed that there is decrease in SLAMF7 expression intensity on PCs from monoclonal gammopathy of undetermined significance (MGUS) cases to MM cases in relapsed/progressive stages of the disease. In addition Ishibashi et al. ${ }^{\left({ }^{(10)}\right.}$ found that serum SSLAMF7 may reflect MM disease progression and could be a useful prognostic indicator in newly diagnosed MM. This is in concordance with our study that illustrates role of CD319 (SLAMF7) as a prognostic marker in PCM patients.

\section{CONCLUSION}

CD319 is a stable immunophenotypic marker with strong expression on PCs and there was highly statistically significant strong positive correlation between CD $319 \%$ and its expression (MFI), CD138/38, and CD56. That proved its role as a diagnostic marker of PCM and can be effectively used as a gating marker in addition to traditional markers. Low expression of CD319 associated with a significant better response to treatment and good prognosis but high expression of CD319 accompanied by bad response to treatment and bad prognosis. This indicates importance of CD319 as a prognostic marker of PCM.

\section{RECOMMENDATIONS}

We recommend to conduct this study on larger sample size for more comprehensive statistical analysis. More studies are required to replace CD138 with CD319 and diagnosis of PCM depends mainly on CD319. Follow up of PCM patients for longer period is needed to verify the prognostic effect of CD319 and its expression. Further studies are required to confirm anti-CD319 (Elotuzumab) as suitable target for immune therapeutic intervention.

\section{REFERENCES}

1. Rajkumar S, Dimopoulos M, Palumbo A et al. (2014): International Myeloma Working Group updated criteria for the diagnosis of multiple myeloma. Lancet Oncol., 15: 538-548.

2. Siegel R, Miller K, Jemal A (2015): Cancer statistics. CA Cancer J Clin., 66:7-30.
3. Frigyesi I, Adolfsson J, Ali M et al. (2014): Robust isolation of malignant plasma cells in multiple myeloma. Blood, 123:1336-43.

4. Rawstron A, Orfao A, Beksac M et al. (2008): European Myeloma Network. Report of the European Myeloma Network on multiparametric flow cytometry in multiple myeloma and related disorders. Haematologica, 93:431-438.

5. NCCN (2021): Multiple Myeloma, Version 3.2021, National Comprehensive Cancer Network (NCCN) Clinical Practice Guidelines in Oncology. https://jnccn.org/ view/journals/jnccn/18/12/articlep1685.xml.

6. Kumar S, Paiva B, Anderson K et al. (2016): IMWG consensus criteria for response and minimal residual disease assesment in multiple myeloma. The Lancet Oncology, 16: 328-346

7. Bouatay A, Hizem S, Youssef B et al. (2013): Myélome multiple: aspect clinique, diagnostic biologique et pronostic. Immunoanalyse \& Biologie Spécialisée, 28(1):30-35.

8. Pojero F, Flores-Montero J, Sanoja L et al. (2015): Utility of CD54, CD229, and CD319 for the identification of plasma cells in patient with clonal plasma cell diseases. Cytometry Part B., 90: 91-100.

9. Aita MHC, Arantes LC, Aita BC et al. (2015): Comparison between immunofixation and electrophoresis for the early detection of relapsed multiple myeloma. Med Lab., 51(6): 359-368.

10. Ishibashi M, Soeda S, Sasaki M et al. (2018): Clinical impact of serum soluble SLAMF7 in multiple myeloma. Oncotarget, 9 (78): 34784-34793.

11. Flores-Montero J, Sanoja-Flores L, Paiva B et al. (2017): Next Generation Flow for highly sensitive and standardized detection of minimal residual disease in multiple myeloma. Leukemia, 31:2094-2103.

12. Sriram H, Ghogale $S$, Subramanian $P$ et al. (2019): Evaluation of CD319 (SLAMF7) as a Novel Gating Marker for Plasma Cells in Flow Cytometric Immunophenotyping of Multiple Myeloma. Clinical Lymphoma, Myeloma and Leukemia, 19(10): 153-158.

13. Veillette A, Guo H (2013): CS1, a SLAM family receptor involved in immune regulation, is a therapeutic target in multiple myeloma. Crit Rev Oncol Hematol., 88: 168-177.

14. Touzeau C, Moreau P, Dumontet C (2017): Monoclonal antibody therapy in multiple myeloma. Leukemia, 31:1039-1047.

15. Soh K, Tario J, Hahn T et al. (2020): CD 319 (SLAMF7) an alternative marker for detecting plasma cells in the presence of daratumumab or elotuzumab. Cytometry, 20: 1-12.

16. Lisenko K, Schönland S, Hegenbart U et al. (2017): Potential therapeutic targets in plasma cell disorders: A flow cytometry study. Cytometry Part B, 92: 145-152. 